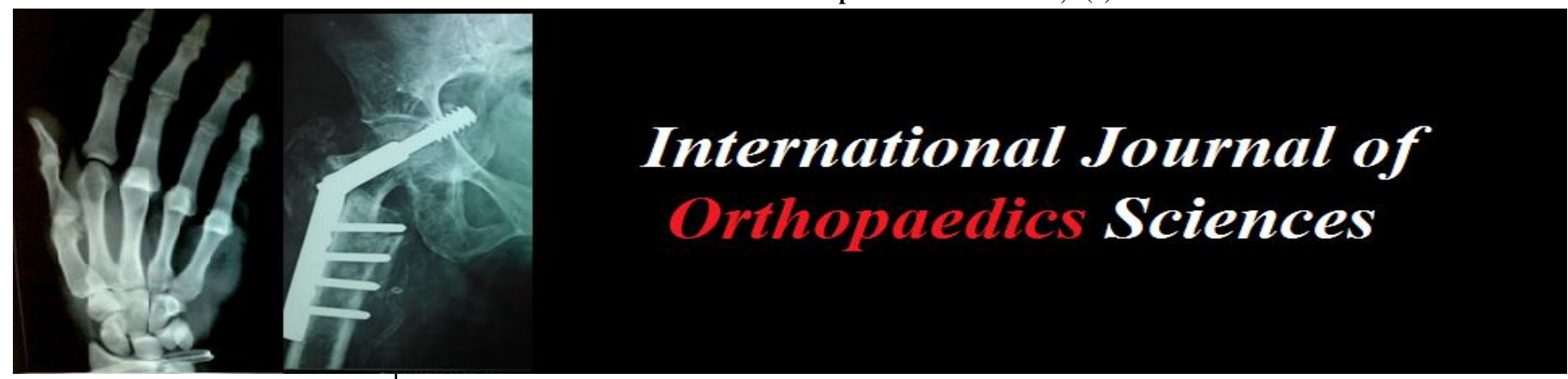

E-ISSN: 2395-1958

P-ISSN: 2706-6630

IJOS 2020; 6(3): 363-366

(C) 2020 IJOS

www.orthopaper.com

Received: 22-05-2020

Accepted: 24-06-2020

\section{Prathap US}

Department of Orthopaedics, HOSMAT Hospital, Magrath Road, Bengaluru, Karnataka, India

Keshava Murthy D

Department of Orthopaedics, HOSMAT Hospital, Magrath Road, Bengaluru, Karnataka, India

Narkhede Yogesh Dilip Department of Orthopaedics, HOSMAT Hospital, Magrath Road, Bengaluru, Karnataka, India

Corresponding Author:

Prathap US

Department of Orthopaedics, HOSMAT Hospital, Magrath Road, Bengaluru, Karnataka, India

\section{Functional outcome of posterior cruciate ligament avulsion injuries treated with open reduction and cannulated cancellous screw fixation}

\author{
Prathap US, Keshava Murthy D and Narkhede Yogesh Dilip
}

DOI: $\underline{\text { https://doi.org/10.22271/ortho.2020.v6.i3f.2225 }}$

\section{Abstract}

Introduction: Isolated bony posterior cruciate ligament (PCL) avulsion fractures are infrequent, estimated to account for $3 \%-20 \%$ of knee ligament injuries. Various surgical techniques have been described for PCL avulsion injuries in the past including alternatives to avoid potentially injurious exposure of popliteal fossa. The purpose of our study was to evaluate functional outcome of PCL avulsion fractures of tibia fixed with open reduction and cannulated cancellous screw.

Material and Methods: Twenty four patients were included in the study. Fixation was done through posteromedial approach using $4 \mathrm{~mm}$ cannulated cancellous screws. Functional outcome was evaluated using Lysholm knee score.

Results: Out of 24 patients 18 were males and 6 were females. The mean age was 31 years. Majority of the patients $(80 \%)$ achieved full functional ROM with no laxity. $8.3 \%$ had an extensor lag and $12 \%$ had grade 1 laxity. The mean preoperative and postoperative Lysholm score were 26.33 and 91 respectively. The difference between the preoperative and the postoperative Lysholm score was statistically significant $(P<0.001)$.

Conclusion: The PCL avulsion injuries from tibia treated with open reduction and CC screw fixation through the posteromedial approach achieves anatomical reduction, restores PCL tension and prevents posterior translation of tibia. The study resulted in excellent functional and radiological outcome.

Keywords: PCL avulsion, lysholm score, burks and schaffer approach

\section{Introduction}

The Posterior Cruciate Ligament (PCL) is the main posterior stabilizer of the knee and limits posterior tibial translation ${ }^{[1]}$. The PCL also plays a role as a central axis controlling and imparting rotational stability to the knee. The most effective management of PCL injuries remains unclear and the natural history of untreated PCL injuries is controversial.

Isolated bony posterior cruciate ligament (PCL) avulsion fractures are infrequent, estimated to account for 3\% - 20\% of knee ligament injuries ${ }^{[1,2]}$. Tibial- sided PCL avulsion fractures are the most common form of isolated bony PCL disruption ${ }^{[3]}$. Avulsion or peel-off PCL injuries at the femoral site have been reported in the literature but are rare ${ }^{[4,5]}$.

The most commonly reported mechanism of injury for an isolated PCL disruption with or without avulsion of a bony fragment is the classic dashboard injury: a posteriorly directed force is applied to the proximal tibia of the knee in flexed position as happens in motorcycle or automobile accidents.

The patient with an acutely injured PCL presents with swelling, pain and inability to bear weight. Ecchymosis or abrasions of the overlying skin and soft tissue are other typical findings, but swelling and painful muscle spasm can mask the two classic tests for detecting a PCL injury, the posterior drawer test and posterior sag sign ${ }^{[6]}$.

It is generally agreed that a displaced or unstable bony avulsion of PCL should be anatomically reduced and rigidly fixed as reported by the previous studies in which excellent functional results were described ${ }^{[7-9]}$.

Various surgical techniques have been described for PCL avulsion injuries in the past including alternatives to avoid potentially injurious exposure of popliteal fossa. The techniques include open reduction and $\mathrm{k}$ wire fixation, cannulated cancellous screw fixation, spiked plate 
and cannulated lag screw fixation ${ }^{[10]}$ and also arthroscopic fixation of PCL injuries.

Arthroscopic fixation is gaining popularity and numerous studies have been published on arthroscopic fixation of PCL injuries. Open reduction and fixation of PCL retains normal ligament and its tension as compared to PCL reconstruction using a graft. ORIF will also confirm fixation of avulsed fracture to its anatomical site.

The purpose of our study was to evaluate functional outcome of PCL avulsion fractures of tibia fixed with open reduction and cannulated cancellous screw.

PCL avulsion injuries from tibia have been classified by White and Patel et al. into 3 groups ${ }^{[11]}$.



Fig 1: classification of PCL avulsion injuries.

Type I fractures are undisplaced.

Type II fractures are "hinged" with superior displacement of only the posterior aspect of the avulsed fragment.

Type III fractures are completely displaced

\section{Materials and Methods}

The study was conducted on patients with isolated PCL avulsion injuries treated with open reduction and fixation with cannulated cancellous screw fixation in the Department of Orthopaedics, HOSMAT Hospital, Bangalore from september 2013 to august 2017.

Approximate sample size -24 .

Method of collection data:

Patients with isolated PCL avulsion fractures who satisfy the inclusion criteria and exclusion criteria will be included in the study. The patients will be evaluated clinically and functionally at the time of examination.

\subsection{Inclusion criteria}

1. Isolated PCL avulsion fractures.

2. Age 15-60 years.

\subsection{Exclusion criteria}

1. Open fractures.

2. Associated with other ligament injuries of knee.

3. Prior surgery on the involved knee.

4. Associated with other fractures of tibia/femur.

\subsection{Surgical Technique}

Anesthesia - Spinal

Position of the patient - Prone

Under tourniquet control, aseptic precaution patient parts are painted and draped. A curvilinear incision taken with horizontal limb just proximal to flexion crease of the knee and vertical limb overlying the medial head of gastrocnemius muscle. Dissection is carried to deep fascial layer and plane is created between medial head of gastrocnemius and semimembranosus. A retractor is placed under the gastrocnemius which is reflected laterally protecting the neurovascular bundle.

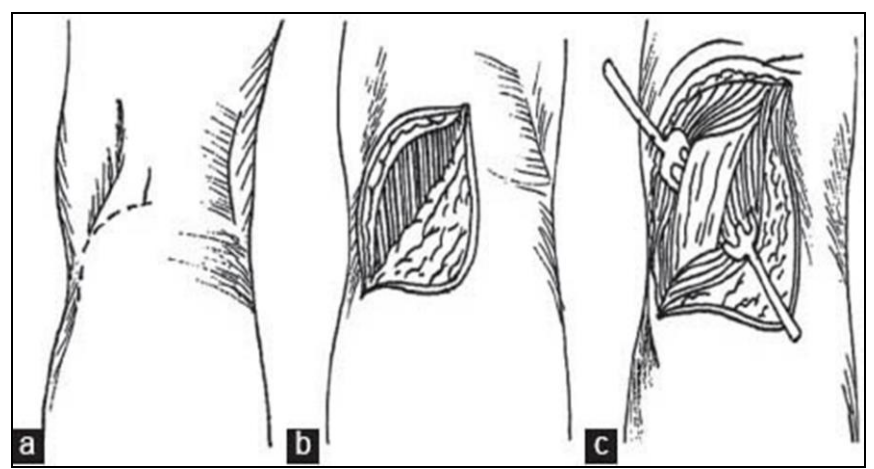

Fig 2: (a) Posteromedial curvilinear incision is made. (b) Superficial dissection, medial head of the gastrocnemius. (c) Reflection of two parts of the medial head of the gastrocnemius, posterior capsule.

With reflection of the gastrocnemius posterior joint capsule is exposed. Occasionally, there is a branch of the middle geniculate artery which lies on the capsule which needs to be ligated or cauterized for hemostasis. The capsule is then incised to expose the avulsed fragment.

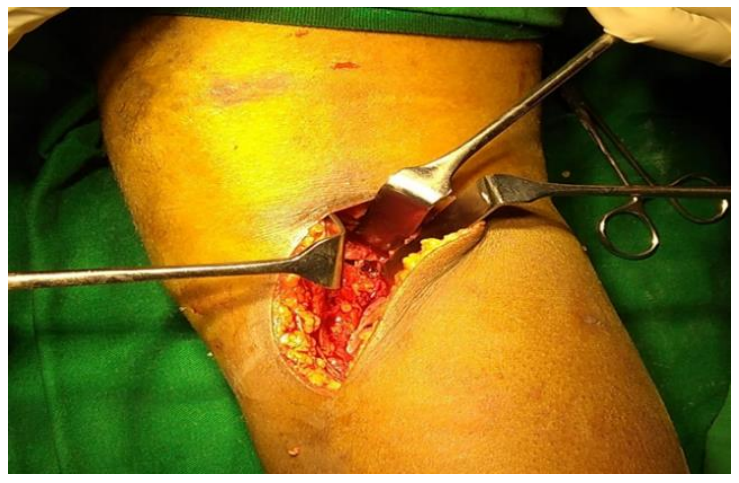

Fig 3: Intraop photo of incision and retraction of medial head of gastrocnemius.

Once the fragment is isolated and fracture pattern is established, the fracture site is irrigated and the clot is debrided. The fracture is reduced and held in place with a $\mathrm{k}$ wire (Fig 3).

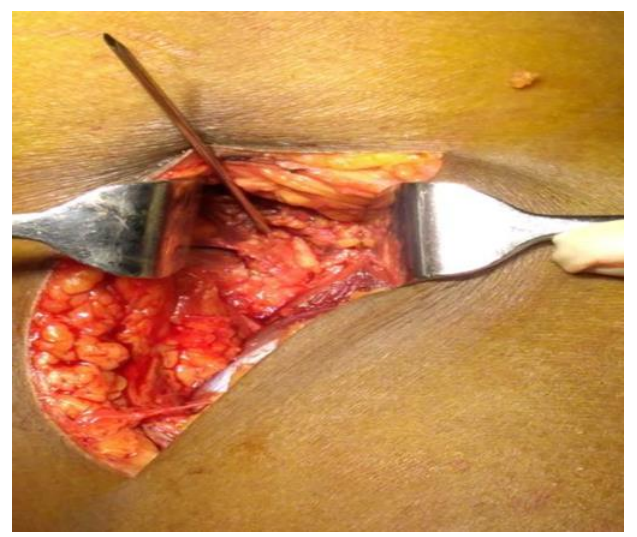

Fig 4: PCL avulsed fragment held using k wire. 
Then drill hole is made using cannulated drill bit and suitable $4 \mathrm{~mm}$ cannulated cancellous screw is fixed and length is checked under c -arm. Usually one screw is sufficient to hold the fragment if not two cannulated cancellous screw may be used (Fig 4, 5).

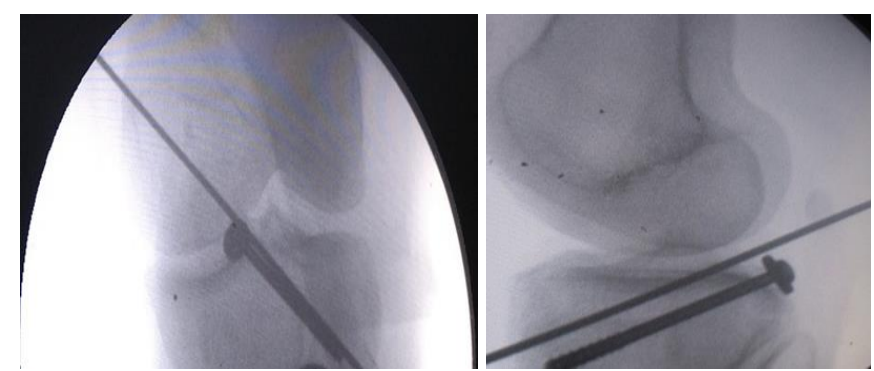

Fig 5: AP and Lateral c-arm images of PCL avulsion fixation.

The tourniquet is then deflated, hemostasis is achieved, and the incision is closed in layers. Sterile dressing is done and then placed into a PCL brace to prevent any further posterior displacement.
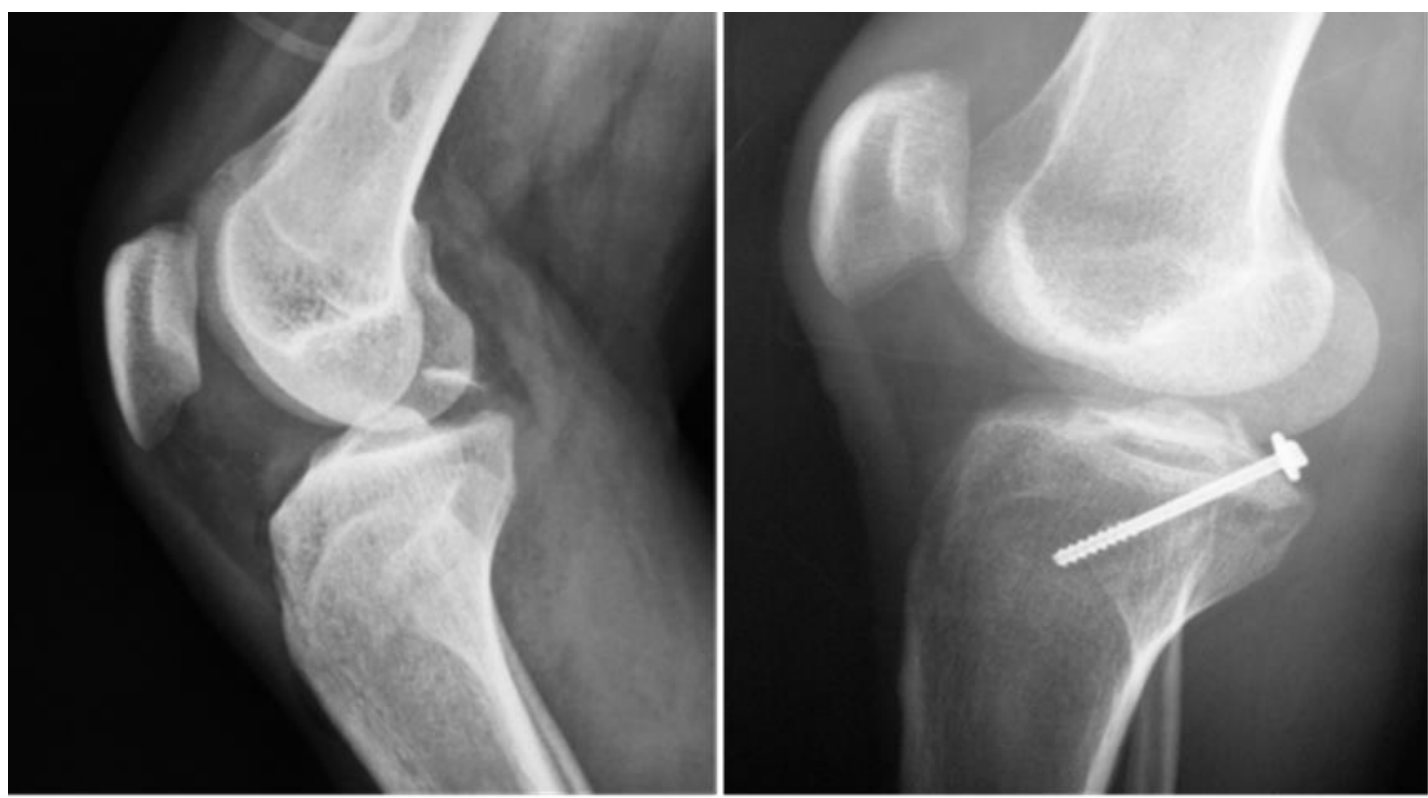

Fig 6: Lateral radiograph of pre-op and post-op PCL avulsion injury.

\section{Results}

The study included 24 patients with PCL avulsion injuries treated with open reduction and cannulated cancellous screw fixation using postero medial approach.

The average age of the patients was 31 years, 18 were males and 6 were females. Most common mode of injury is Road
Postoperatively all patients mobilised with non weight bearing ambulation with walker for 4 weeks. Later physiotherapy is started active range of movements and quadriceps strengthening exercises till the patient returns to pre injury status.

Tools: Patients were then taken up for open reduction and fixation with $4 \mathrm{~mm}$ cannulated cancellous screw for PCL avulsion fractures from tibia. Postoperative protocol was followed. All patients completed questionnaires to determine Lysholm knee scoring scale and data were obtained prior to surgery and at the time of followup.

As Lysholm knee scoring scale considers both subjective and objective evaluation, it was considered for the study. The scoring scale carries maximum points for instability and pain, which affects the patients satisfactory level. The maximum score in Lysholm knee score is 100. A score of 100 means no symptom or no disability. The scores are categorised as-

EXCELLENT: 91-100

GOOD: $84-90$

FAIR: $65-83$

POOR: $<64$

Table 1: Comparision of pre op and post op Lysholm score by mean and stadard deviation.

\begin{tabular}{|c|c|c|}
\hline Lysholm score group & Mean & SD \\
\hline Pre op & 26.33 & 2.12 \\
\hline Post op & 91 & 3.36 \\
\hline
\end{tabular}

traffic accident (71\%) followed by domestic fall (17\%) and sports related (12\%). The mean pre-op Lysholm score was 26.33 which improved postoperatively to 91 (Table 1). 71\% of patients achieved full ROM at the time of followup and $88 \%$ had grade zero laxity (Table $2 \& 3$ ). followup.

\begin{tabular}{|c|c|c|c|}
\hline Sl no & Range of motion(degree) & Frequency & Percentage \\
\hline 1 & $130-140$ (full) & 17 & 70.83 \\
\hline 2 & $120-130$ & 5 & 20.83 \\
\hline 3 & Upto 120 with lag & 2 & 8.33 \\
\hline
\end{tabular}

Table 2: Distribution of samples by Range of Motion at the time of 西 Table 3: Distribution of samples by laxity grading by posterior
drawer test at the time of followup.

\begin{tabular}{|c|c|c|c|}
\hline Grade & Amount of laxity $(\mathbf{m m})$ & Frequency & Percentage \\
\hline 0 & Nil & 21 & 87.5 \\
\hline 1 & $<5$ & 3 & 12.5 \\
\hline 2 & $6-10$ & 0 & 0 \\
\hline 3 & $>10$ & 0 & 0 \\
\hline
\end{tabular}


Table 4: Distribution of samples by return to preinjury status.

\begin{tabular}{|c|c|c|}
\hline Preinjury status & Frequency & Percentage \\
\hline Returned & 22 & 91.66 \\
\hline Not returned & 2 & 08.33 \\
\hline
\end{tabular}

\section{Discussion}

The PCL avulsion fractures from tibia must be treated surgically with either open reduction and internal fixation or an arthroscopic fixation. The arthroscopic method is an ideal method provided the necessary expertise and equipments are available. This method is less invasive and early rehabilitation can be started. Hence patient compliance is more.

Despite being ideal method of fixation, Kim et al. ${ }^{[12]}$ believed that the technique of arthroscopy-assisted reduction and fixation was difficult and had a steep learning curve. In our experience a similar fixation can also be achieved by open exposure through the posteromedial approach. Also, open reduction and internal fixation achieves anatomical reduction, restores PCL tension and prevents posterior translation of tibia.

Mechanism of injury - Most common mechanism of PCL avulsion injuries is RTA followed by sports related injuries and domestic fall. $71 \%$ of the cases in our study had RTA. Seitz et al. ${ }^{[6]}$ in his study also found $87 \%$ to be dashboard type with all these patients having pretibial ecchymosis.

Approach - The standard classical approach described by Abbott et al. ${ }^{[13]}$ was extensive, time consuming and associated with the risk of damage to neurovascular structures. We used modified Burks and Schaffer ${ }^{14}$ approach for fixation of PCL avulsion fractures, it simplified the procedure and did not require either division of medial head of gastrocnemius as we retract the medial head of gastrocnemius along with neurovascular bundle. The technique needs a short learning curve, minimal dissection, less time consuming and no risk of damage to neurovascular structures. It also provides direct tibial PCL access, which made this approach the best option for fixation of bony PCL avulsions. The post-operative rehabilitation was accelerated and excellent functional results were gained.

Range of Movements (ROM) - Majority of the patients who underwent $\mathrm{CC}$ screw fixation of PCL tibial avulsion fracture had full ROM at the time of followup. In our study $71 \%$ of the patients had full ROM at the time of follow up, $21 \%$ of patients had terminal restriction of flexion. $8 \%$ of the patients had extensor lag of 10 degree which is due to postoperative non-compliance and discontinued physiotherapy in the form of active quadriceps strengthening exercises. This suggest the importance of postop rehabilitation in cases of PCL avulsion fixed using CC screws. Milind V Ingle et al. ${ }^{[15]}$ in their study also had got similar results with $66 \%$ of the patients achieving full ROM. 25\% patients had ROM between 130-140 degree(terminal restriction) which was clinically acceptable to them. The reduced ROM in remaining $10 \%$ of cases could be due to non-compliance and apprehension for physiotherapy.

\section{Conclusion}

Open reduction and internal fixation with Cannulated Cancellous screws for PCL avulsion injuries from tibia is one of the excellent techniques of surgery. It is technically easier and reproducible and does not require specialised equipment. It is best done using modified Burks and Schaffer (posteromedial) approach which is less time consuming and needs minimal dissection. It has relatively short learning curve. It is possible to get anatomical reduction and fixation for complete restoration of PCL function with open reduction and internal fixation.

The morbidity as well as the speed of recovery are comparable to that following arthroscopic fixation. It can be performed in centres where arthroscopic equipments and expertise are not available. Arthroscopic repair or fixation also yields good results but can be used only when the avulsed fragment is small. However arthroscopic technique is associated with significant learning curve, In addition, type 1 avulsion injuries can be managed conservatively and may not require arthroscopic reduction and stabilization.

\section{References}

1. Kohli S, Vishwakarma N, Chauhan S, Salgotra K. Functional evaluation of fixation for PCL bony avulsion fractures using Burk and Schaffer's approach. International Journal of Orthopaedics Sciences. 2017; 3(1):735-739.

2. Veltri DM, Warren RF. Isolated and Combined Posterior Cruciate Ligament Injuries. J Am Acad Orthop Surg. 1993; 1(2):67-75.

3. Katsman A, Strauss EJ, Campbel KA, Alaia MJ. Posterior Cruciate Ligament Avulsion Fractures. Curr Rev Musculoskelet Med. 2018; 11:503-509

4. Giordano BD, Dehaven KE, Maloney MD. Acute femoral "peel-off" tears of the posterior cruciate ligament: technique for arthroscopic anatomical repair. Am J Orthop. 2011; 40(5):226-232,

5. Park IS, Kim SJ. Arthroscopic fixation of avulsion of the posterior cruciate ligament from femoral insertion. Arthroscopy. 2005; 21(11):1397.

6. Seitz H, Schlenz I, Pajenda G, Vecsei V. Tibial avulsion fracture of the posterior cruciate ligament: K-wire or screw fixation? A retrospective study of 26 patients. Arch Orthop Trauma Surg. 1997; 116:275-8.

7. Meyers MH. Isolated avulsion of the tibial attachment of the posterior cruciate ligament of the knee. J Bone Joint Surg Am. 1975; 57:669-672.

8. Lee HG. Avulsion fracture of the tibial attachment of the cruciate ligaments. J Bone Joint Surg. 1937; 19:460-468.

9. Torisu T. Isolated avulsion fracture of the tibial attachment of the posterior cruciate ligament. J Bone Joint Surg Am. 1977; 59:68-72.

10. Chen W, Luo W, Chen Z, Jiang Y. Treatment of posterior cruciate ligament avulsion fractures of the tibia using a toothed plate and hollow lag screw. Singapore Med J. 2016; 57(1):39-44.

11. White EA, Patel DB, Matcuk GR, Forrester DM, Lundquist RB, Hatch GF et al. Cruciate ligament avulsion fractures: Anatomy, biomechanics, injury patterns, and approach to management. Emerg Radiol. 2013; 20(5):429-40.

12. Kim SJ, Shin SJ, Choi NH, Cho SK. Arthroscopically assisted treatment of avulsion fractures of the posterior cruciate ligament from the tibia. J Bone Joint Surg. 2001; 83-A:698-708.

13. Abbott LC, Carpenter WF. Surgical approaches to the knee joint. J Bone Joint Surg. 1945; 27:277-310

14. Burks RT, Schaffer JT. A simplified approach to the tibial attachment of the posterior cruciate ligament. Clin Orthop. 1990; 254:216-9.

15. Ingle MV, Bhalotia AP, Chandele V, Gade S, KoichadeMR. Functional outcome of bony PCL avulsions fixed with Mini open technique. International Journal of Orthopaedics Sciences. 2017; 3(1):81-84. 